\title{
Tryptophan Released From Mother's Milk Has Antioxidant Properties
}

\author{
APOLLINAIRE TSOPMO, BILL W. DIEHL-JONES, ROTIMI E. ALUKO, DAVID D. KITTS, INGRID ELISIA,
} AND JAMES K. FRIEL

\begin{abstract}
Chemistry Department [A.T.], Carleton University, Ottawa, Ontario, Canada K1S 5B6; Nursing and Biological Sciences [B.W.D.-J.], Department of Human Nutritional Sciences [R.E.A., J.K.F.], and Department of Pediatrics [J.K.F.], University of Manitoba, Winnipeg, Manitoba, Canada R3T 6C5; Department of Food, Nutrition, and Health [D.D.K., I.E.], University of British Columbia, Vancouver,
\end{abstract} British Columbia, Canada V6T 1Z4

\begin{abstract}
Bioactive factors in human milk (HM) are crucial to the health of newborns, especially preterm infants. These compounds assist in reducing the oxidative stress that may occur as a result of combined exposure to supplemental oxygen and immature physiologic defenses. To identify the components in HM that contribute to its greater resistance to oxidative stress compared with infant formulae, enzymatic hydrolysates of HM were prepared, ultrafiltered, separated, and analyzed for antioxidant potential. The antioxidant activity $[\mu \mathrm{M}$ Trolox equivalent $(\mathrm{TE} / \mathrm{g})]$ of nondigested milk, whole digested milk, and derived ultrafiltrates were 80.4 \pm 13.3, $159.0 \pm$ 5.6, and $127.4 \pm 3.1$, respectively. An HPLC fraction denoted as fraction 23 (5274 $\pm 630 \mu \mathrm{M}$ TE/g) was obtained and its constituents identified as tryptophan (Trp), peptides HNPI, and PLAPQA. Scavenging activity was not observed for PLAPQA, whereas moderate activity was associated with HNPI $(144 \pm 10.7 \mu \mathrm{M}$ TE/g $)$ and very high activity to $\operatorname{Trp}(7986 \pm 468 \mu \mathrm{M}$ TE/g). Trp addition to HM and two infant formulas significantly increased formulae antioxidant properties. Trp appeared to be a powerful free radical scavenger naturally present in HM. Its antioxidant effects and potential application in the diets of infants, particularly preterm, must be examined further. (Pediatr Res 66: 614-618, 2009)
\end{abstract}

$\mathrm{O}$ xidative stress is implicated as an underlying factor in many pathologic processes of human health disorders (1). Birth is a physiologic process associated with enhanced stress due to the transition of the fetus from a relatively hypoxic condition in the womb (2) to a relatively hyperoxic environment during early neonatal life (3). Premature infants, in particular, are often exposed to supplemental oxygen concentrations as high as $95 \%(4,5)$; the result of which is the overproduction of reactive oxygen species (ROS). Numerous health conditions among preterm infants that are linked to oxidative stress include bronchopulmonary dysplasia, retinopathy of prematurity, intraventricular hemorrhage, necrotizing enterocolitis, and renal failure; all of which can lead to subsequent long-term complications in the physical and neurologic development of the child $(5,6)$. The term "oxygen radical dis-

Received May 4, 2009; accepted August 25, 2009.

Correspondence: James K. Friel, PhD, Room 203, Richardson Centre for Functional Foods and Nutraceuticals, 196 Innovation Drive, University of Manitoba, Winnipeg, MB, Canada R3T 6C5; e-mail: frielj@cc.umanitoba.ca

Supported by the Advanced Foods and Materials Network (AFMNet) Centre of Excellence and the Manitoba Institute of Child Health (MICH). ease" was proposed to represent all the conditions affecting preterm infants that may contribute to neonatal morbidity (7).

ROS can react with polyunsaturated fatty acids of cell membranes, proteins, polysaccharides, and DNA resulting in functional alterations within the cell and subsequent tissue/ cellular injury (8). Several mechanisms lead to ROS overproduction in the perinatal period, including ischemiareperfusion, increased levels of free iron, and products of the arachidonic acid cascade (9). ROS generation in vivo is controlled by the action of antioxidant enzymes, such as superoxide dismutase (SOD), catalase (CAT), and glutathione peroxidase $(\mathrm{GPx})$, and the presence of nonenzymatic antioxidants, such as vitamins $\mathrm{C}$ and $\mathrm{E}$ and other bioactive agents $(2,10)$.

Human milk (HM) is recognized as the primary and optimal food for newborns. The importance of nonenzymatic, bioactive antioxidants in $\mathrm{HM}$ is important because endogenous infant enzyme maturation does not occur before 28 -wk of gestation and the transfer of vitamin $\mathrm{E}$ from the mother to the fetus occurs primarily during the last trimester of pregnancy $(11,12)$. Thus, there are limitations to the development of the antioxidant defense system in both premature and full-term newborns $(9,10)$, which leaves them susceptible to the adverse effects of oxidative stress (2). We postulate that HM with bioactive agents is crucial to newborns, especially preterm babies, and will assist in reducing oxidative stress while physiologic defenses are underdeveloped. For example, HM itself contains antioxidant enzymes SOD, CAT, and GPx, which eliminate superoxide anion, hydrogen peroxide, and hydroperoxide, respectively, whereas vitamins $\mathrm{C}$ and $\mathrm{E}$ provide direct free radical-scavenging benefits; as well lactoferrin binds iron and prevents the generation of ROS through the Fenton reaction $(2,13)$. Although HM has been found to have higher antioxidant content than infant formula, the complete list of active antioxidant compounds in HM is presently unknown $(14,15)$. The identification of these naturally occurring bioactives in HM would further enhance our understand-

Abbreviations: AAPH, 2,2' -azobis (2-amidinopropane) dihydrochloride; CAT, catalase; HM, human milk; ORAC, oxygen radical absorbance capacity; ROS, reactive oxygen species; SOD, superoxide dismutase; TE, Trolox equivalent; TFA, trifluoroacetic acid; Trp, tryptophan 
ing of the biochemistry that explains the antioxidant capabilities of mother's milk.

In this study, we modified an in vitro digestion procedure to process mother's milk and recovered crude digests that were further separated into more distinct fractions by column chromatography, and subsequently characterized for antioxidant activity using the oxygen radical absorbance capacity (ORAC) assay. Efforts were made to isolate and characterize the individual components of the HM digest fractions that exhibited antioxidant activity toward the peroxyl radical generated by thermal lysis of 2,2'-Azobis (2-amidinopropane) dihydrochloride $(\mathrm{AAPH})$.

\section{METHODS}

Milk samples. HM samples were obtained from three volunteer mothers who were breastfeeding their infants. The milk was collected using a mechanical breast pump (Ameda-Egnel Lincolnshire, IL) by each lactating mother during the first $4 \mathrm{mo}$ and stored at $-20^{\circ} \mathrm{C}$. All samples were transported to our laboratory on dry ice. Once in our laboratory, samples from each mother were thawed at room temperature, combined, freeze-dried, and then stored at $-80^{\circ} \mathrm{C}$ until ready for analysis.

Chemicals and reagents. Pepsin P-7000, pancreatin P1750, bile salts B333, 6-hydroxy-2,5,7,8-tetramethylchroman-2-carboxylic acid (Trolox), 2,2'-azobis (2-amidinopropane) dihydrochloride (AAPH), trifluoroacetic acid (TFA), and L-tryptophan (Trp) were purchased from Sigma Chemical Co. Aldrich Canada Ltd (Oakville, Ontario). Methanol and acetonitrile HPLC grade and fluorescein were purchased from Fisher Scientific Canada (Ottawa, Ontario). High-purity water was produced in the laboratory by an Alpha-Q system (Millipore, Marlborough, MA).

Digestion of milk samples. Freeze-dried HM was rehydrated immediately before conducting the experiments. Because total solids in HM account for approximately $13 \%$ (16), we used $130 \mathrm{~g}$ of freeze-dried HM powder and diluted this to $1 \mathrm{~L}$ using deionized water before conducting the two-step digestion experiments. Digestion of HM was performed using modified procedures of Etcheverry et al. (17) and Jovani et al. (18). In both these studies, a pH 2.0 was used to approximate the conditions present during adult gastric digestion. We used $\mathrm{pH} 4.0$ and 6.0 for the two-step digestion procedure. This change in protocol was to more closely approximate digestion in the premature infant gut and based on a study by Omari and Davidson (19), whereby these $\mathrm{pH}$ values were recorded in preterm infants using a fourchannel probe to measure the esophageal and intragastric changes in $\mathrm{pH}$ after feeding. The $\mathrm{pH}$ of the reconstituted $\mathrm{HM}$ was adjusted to 5.5 with $1 \mathrm{M} \mathrm{HCl}$, $76 \mathrm{~mL}$ of pepsin $(4 \mathrm{~g} / 100 \mathrm{~mL}$ of $0.1 \mathrm{M} \mathrm{HCl})$ added, and final $\mathrm{pH}$ adjusted to 4.0. The sample was incubated for $30 \mathrm{~min}$ at $37^{\circ} \mathrm{C}$ with gentle shaking at 100 rpm in a MaxQ 4000 incubator (Barnstead Lab-Line), after which the $\mathrm{pH}$ was adjusted to 6.0 and the sample was further incubated for another $30 \mathrm{~min}$. After peptic digestion, $380 \mathrm{~mL}$ of a $0.1 \mathrm{M} \mathrm{NaHCO}_{3}$ solution containing pancreatin $(0.8 \mathrm{~g} / 400 \mathrm{~mL})$ and bile salts $(4.8 \mathrm{~g} / 400 \mathrm{~mL})$ were added. The solution was adjusted to $\mathrm{pH} 7.0$ with $1 \mathrm{M} \mathrm{NaHCO}_{3}$ and incubation was carried out for $2 \mathrm{~h}$ at $37^{\circ} \mathrm{C}$. After incubation, the sample solution was heated in a water bath at $90^{\circ} \mathrm{C}$ for 15 min to inactivate pancreatin enzymes.

Ultrafiltration and HPLC separations. The digested sample was transferred to a separatory funnel and stored for $5 \mathrm{~h}$ at $4{ }^{\circ} \mathrm{C}$ to separate the lipid and aqueous phases. An Amicon stirred cell (unit model 8400) containing a 3000 Da molecular weight cutoff membrane (Millipore, Billerica, MA) was used to separate the aqueous phase into low molecular weight $(<3000 \mathrm{Da})$ peptide fraction (permeate). All digested samples were freeze dried and stored at $-80^{\circ} \mathrm{C}$.

HPLC separation of the 3000 Da membrane permeate was performed using a 600E Multisolvent Delivery System equipped with a $5 \mathrm{~mL}$ manual sample loop, $100 \mu \mathrm{L}$ pump head, 717 autosampler, 996 PDA detector, and fraction collector III (Waters Corporation; Milford, MA). The column used was Waters Symmetry $300 \mathrm{C} 18(5 \mu \mathrm{m} 19 \times 250 \mathrm{~mm})$. Solvent A was $0.05 \% \mathrm{TFA}$ in water and solvent B contained $0.05 \%$ TFA in acetonitrile. The HM ultrafiltered permeate $(150 \mathrm{mg} / \mathrm{mL})$ in solvent A was filtered on $0.45-\mu \mathrm{m}$ membrane and $5 \mathrm{~mL}$ were injected onto the HPLC column. A linear gradient of solvent $\mathrm{B}$ ranging from 0 to $70 \%$ in solvent A during $60 \mathrm{~min}$ at a flow rate of $6 \mathrm{~mL} / \mathrm{min}$ was used to separate peptides. Fractions were collected every $\min (6 \mathrm{~mL})$, individually freeze dried and used for the ORAC assay.

Oxygen radical absorbance capacity of digested HM fractions. The assay was performed according to the literature $(20,21)$. An FLx800 microplate fluorescence reader (Bio-Tek Instruments, Inc., Winooski, VT) with fluores- cence filters (excitation 485/20 nm, emission 528/20 nm) was used and run using the KC4 software. Five Trolox concentrations between 6.25 and $100 \mu \mathrm{M}$ were used for calibration curve. The antioxidant control was rutin trihydrate $(20 \mu \mathrm{M})$. Two concentrations (312.50 and $78.10 \mu \mathrm{g} / \mathrm{mL}$ ) of crude HM samples and two concentrations (26.00 and $13.00 \mu \mathrm{g} / \mathrm{mL}$ ) of HPLC fractions were used for each assay run. Data were processed as previously reported (21) and the unit of measurement expressed as Trolox equivalents (TE).

ORAC of supplemented HM and formulae. The effect of added Trp on the endogenous antioxidant potential of HM and milk formula was tested with a mature HM sample and two infant formulae to determine any potential matrix effects. The HM was obtained from an additional volunteer mother and the formulae were Earth's Best Organic (EBO) (Heinz, Pittsburg, PA) and Similac Alimentum Advance (SAA) (Abbott Laboratories, Montreal, PQ) purchased locally in Winnipeg, Manitoba, Canada. The formulae diluted in water according to the manufactures' instructions and thawed HM were nondigested. All samples were then diluted 125 and 250 times in phosphate buffer before ORAC assays were performed. The final Trp concentration in diluted samples was adjusted to $10.2 \mu \mathrm{M}$, which allowed a detectable ORAC reading within our calibration curve.

Identification by $\mathbf{L C}$-MS/MS. Compounds in fraction 23 were identified using an ultra Performance Acquity UPLC system coupled to a Quattro micro API 2000 MS/MS mass spectrometer both from Waters Corporation (both from Waters Corporation, Milford, MA). The UPLC included Acquity autosampler, binary pumps, and Acquity PDA detector (214 and $280 \mathrm{~nm})$. Chromatography was performed using an Acquity BEH C18 column $(50 \times$ $2.1 \mathrm{~mm} ; 1.7 \mu \mathrm{m}$; Waters Corp). The mobile phase was composed of A: $0.1 \%$ formic acid in water and B: $0.1 \%$ formic acid in acetonitrile. A linear gradient from 0 to $20 \% \mathrm{~B}$, delivered at $0.2 \mathrm{~mL} / \mathrm{min}$ during $50 \mathrm{~min}$, was used and the eluates were automatically transferred to the MS system.

The mass spectrometer was operated with an ESCi probe. MS regular scan analysis was performed in electrospray-positive mode: capillary voltage 3.5 $\mathrm{kV}$, source temperature $100^{\circ} \mathrm{C}$, desolvation temperature $200^{\circ} \mathrm{C}$, cone gas 50 $\mathrm{L} / \mathrm{h}$, desolvation nitrogen gas $500 \mathrm{~L} / \mathrm{h}$, and electron multiplier $650 \mathrm{~V}$. Daughter and parent spectra were obtained with a collision energy of $25 \mathrm{eV}$. Data were processed through MassLynx software (version 4.1, Waters Corp). De novo peptides sequencing was performed on MS/MS data using PepSeq software (version 1.2 available at http://www.kuleuven.ac.be/rega/medchem/ pepseq.htm).

Analysis of tryptophan in infant plasma and peptide fraction 23. A blood sample was collected in EDTA-coated tubes from a 1-mo-old breastfed infant in a concurrent study. The sample was centrifuged $\left(3000 \times \mathrm{g}\right.$ at $\left.4^{\circ} \mathrm{C}\right)$ to separate plasma and red blood cells. One hundred microliter of $10 \%$ trichloroacetic acid (TCA) solution was added to $100 \mu \mathrm{L}$ of plasma and the mixture was centrifuged at $10,000 \mathrm{rpm}$ for $10 \mathrm{~min}$ at room temperature. The sample was filtered on a $0.45-\mu \mathrm{m}$ Millipore membrane and $10 \mu \mathrm{L}$ was analyzed using UPLC-MS/MS as described earlier.

Determination of Trp content of fraction 23 and undigested HM was performed by Advanced Protein Technology Centre, Ontario, Canada. Briefly, samples were reconstituted in water and digested with $4 \mathrm{M}$ methanesulfonic acid followed by PITC derivatization. Trp was subsequently separated in reverse-phase Acquity UPLC BEH column $(2.1 \mathrm{~mm} \times 10 \mathrm{~cm})$ at $48^{\circ} \mathrm{C}$ and detected at $254 \mathrm{~nm}$.

Statistical analysis and ethics. All data are expressed as mean \pm SD from replicates analyzed in triplicate. One-way ANOVA was used to compare ORAC values. Statistical significance was set to $p<0.05$. All statistics were completed with SPSSx (Version 16, 2007, Chicago, IL). All procedures received ethical approval by the University of Manitoba Ethics Research Review Board. For milk analysis, the review board did not require informed consent as the milk was discarded and any identifying features were removed. For blood analysis, informed consent was obtained from each mother.

\section{RESULTS}

Radical-scavenging activity of digested human milk, recovered fractions, and tryptophan. The antioxidant activities of HM and different fractions recovered from in vitro digested HM samples, using the ORAC assay, are reported in Table 1. With the exception of the lipid fraction, all isolated fractions possessed peroxyl radical-scavenging activity that was higher than that observed from the undigested whole milk sample (HM). After digestion of HM with pepsin plus pancreatin-bile salts and subsequent fractionation, the ORAC value of a low molecular weight ( $<3000 \mathrm{Da}$ ) fraction was $127.4 \pm 3.1 \mu \mathrm{M} \mathrm{TE} / \mathrm{g}$ of the 
Table 1. ORAC values of digested pepsin and pepsin-pancreatin digested HM fractions

\begin{tabular}{lc}
\hline \multicolumn{1}{c}{ Sample } & ORAC $(\mu \mathrm{M}$ Trolox/g $)$ \\
\hline HM & $80.4 \pm 13.3^{*}$ \\
HM P + P (lipid) & $49.8 \pm 2.5 \dagger$ \\
HM Pepsin & $110.8 \pm 5.4 \ddagger$ \\
HM Pepsin $>3000 \mathrm{Da}$ & $200.1 \pm 3.9 \S$ \\
HM Pepsin $<3000 \mathrm{Da}$ & $48.6 \pm 2.5 \dagger$ \\
HM P + P & $159.0 \pm 5.6 \|$ \\
HM P + P $>3000 \mathrm{Da}$ & $212.3 \pm 11.1 \S$ \\
HM P + P <3000 Da & $127.4 \pm 3.1 \Phi$ \\
\hline
\end{tabular}

ORAC: mean \pm SD.

* Values in the column with different symbols indicate significant differences $(p<0.05)$.

$\dagger$ HM P + P (lipid): lipid from pepsin/pancreatin digested HM.

\$ HM Pepsin: pepsin digested.

$\S$ HM Pepsin >3000 Da: pepsin digest retained on 3000 Da membrane.

| HM Pepsin <3000: pepsin digest, permeate from 3000 Da membrane.

If HM P + P <3000 Da: pepsin/pancreatin digest, permeate from $3000 \mathrm{Da}$ membrane.

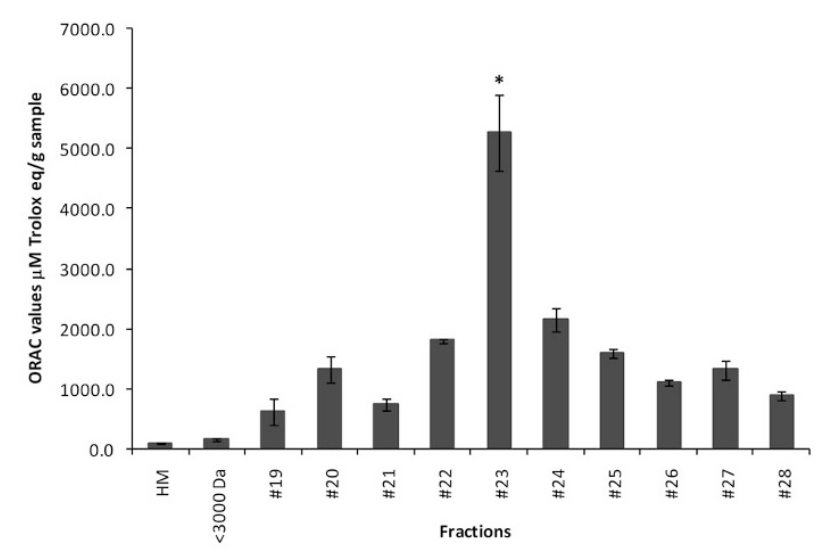

Figure 1. ORAC values (mean $\pm \mathrm{SD}$ ) of HM, ultrafiltered permeate $(<3000)$ and its fractions derived from HPLC. Statistical significance $* p<$ 0.01 compared with all other fractions.

freeze-dried sample. We concentrated on this fraction because it would likely contain molecules that are potentially less susceptible to further digestion and may have higher bioavailability, as they may be capable of crossing neonatal intestinal cell barriers.

To identify the fraction of the digested HM sample with the highest possible bioactivity, the low molecular weight fraction was subjected to reverse-phase HPLC separation, as described in the Methods section. Fifty-five fractions were collected, freeze dried, and evaluated for antioxidant activity using the ORAC assay. The most potent fraction was found to be fraction 23 (Fig. 1), with an ORAC value of $5274 \pm 630 \mu \mathrm{M}$ $\mathrm{TE} / \mathrm{g}$ of freeze-dried sample. The ORAC value of this fraction was markedly higher than both that of undigested HM (80.4 \pm $13.3 \mu \mathrm{M} \mathrm{TE} / \mathrm{g}$ ) and the crude fraction obtained after digestion but before HPLC separation $(127.4 \pm 3.1 \mu \mathrm{M} \mathrm{TE} / \mathrm{g})$. Trp was identified and purchased from Sigma Chemical Co. Aldrich Canada Ltd for further testing. Two peptides identified specifically as HNPI (peak 2) and PLAPQA (peak 4) in fraction 23 were synthesized by GenWay Biotech, Inc (San Diego, CA) and tested for ORAC-scavenging activity. HNPI was

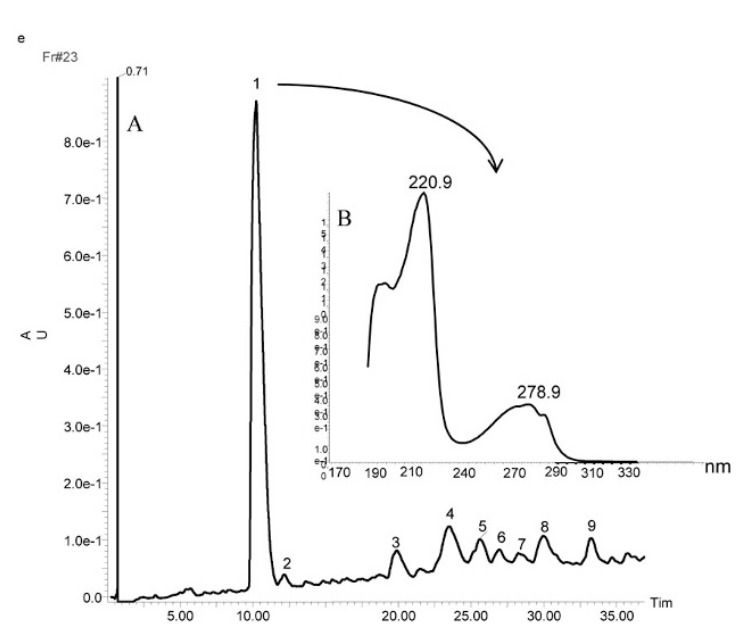

Figure 2. UV chromatogram $(214 \mathrm{~nm})$ of fraction $23(A)$ and online UV spectrum $(B)$ from Acquity UPLC system. Peaks: $1=$ Trp; $2=$ HNPI; $4=$ PLAPQA.

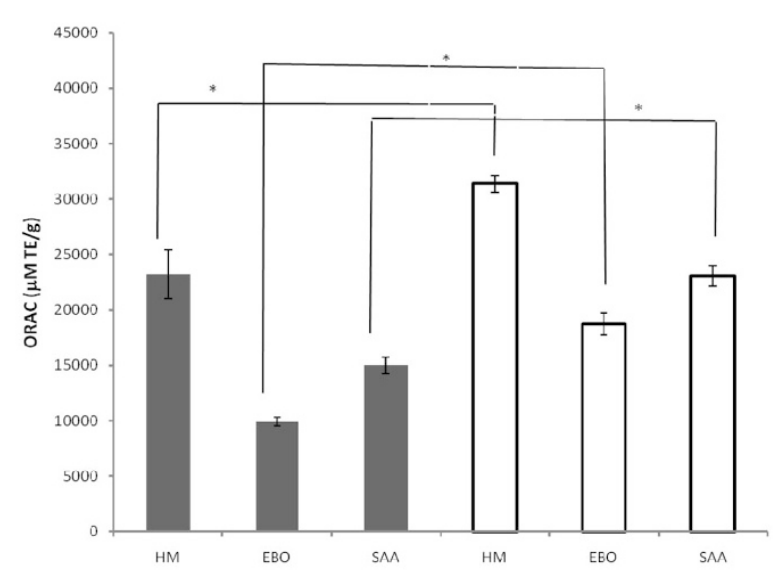

Figure 3. ORAC values of nonsupplemented (black) and supplemented milk (white) with $10.2 \mu \mathrm{M}$ Trp. HM: human milk, EBO, Earth's Best Organic; SAA, Similac Alimentum Advance. Statistical significance $* p<0.01$.

found to have an ORAC value of $114.0 \pm 10.7 \mu \mathrm{M}$ TE/g, whereas no ORAC activity could be detected for PLAPQA.

Identification of compounds in fraction 23. Fraction 23 was analyzed by reverse-phase HPLC coupled to Quattro Micro API electrospray mass spectrometry and the UV chromatogram at $214 \mathrm{~nm}$ is shown in Figure 2. One major peak with a retention time (RT) of $9.47 \mathrm{~min}$ (here referred to as compound 1) and approximately eight other smaller peaks were identified on the chromatogram. Compound 1 was also present at $280 \mathrm{~nm}$, indicating the possible presence of Trp, tyrosine (Tyr), or phenylalanine (Phe), three amino acids that contain an aromatic ring and absorb at $280 \mathrm{~nm}$. The UV spectrum of compound 1 had an absorption maximum at 279 $\mathrm{nm}$, a side peak at $288 \mathrm{~nm}$, and a shoulder at $271 \mathrm{~nm}$; which is common to that reported for Trp (22). From the Quattro MS/MS analysis, the molecular peak was observed at $\mathrm{m} / \mathrm{z}$ 205.3248 for compound 1 , which is identical to the mass of Trp. To confirm the nature of compound 1, a standard L-Trp was analyzed concomitantly with fraction 23 . RTs, UV chromatograms/spectra, and MS data were all identical. From this information, we concluded that compound 1 is free Trp. 
De novo peptide sequencing using the PepSeQ software version 1.2 was performed to determine the sequence of other peaks in peptide fraction 23. Chromatography peaks 2 and 4 were identified as peptides HNPI and PLAQPA, respectively. They are likely derived from human $\beta$-casein subunits and have been reported before from digested HM samples (23). MS/MS data of peaks 5 to 9 showed that they were peptides composed of five to nine amino acids. The final amino acid sequences of these peptides have not yet been determined.

Antioxidant activity of fraction 23 is due to tryptophan. We evaluated the antioxidant activity of fractions 20 to 26 and found that respective ORAC values for these peptides were proportional to the content of Trp based on the area from the UV chromatograms. We also evaluated the antioxidant activity of fresh mother's milk and two infant formulas, EBO and SAA, both before and after supplementation with $10.2 \mu \mathrm{M}$ Trp (Fig. 3).

Tryptophan in infant plasma. A plasma sample from a 1-mo-old breastfed infant in a concurrent study was analyzed by UPLC-MS/MS. The presence of Trp in neonate plasma was confirmed by comparison of RT, UV chromatogram, and MS data with that of fraction 23 and standard Trp.

Among a few selected peptide fractions, fraction 23 was found to contain the highest Trp content at $7.43 \%$ of dried fraction. This level of Trp was approximately 50 times more concentrated than undigested HM, which contained $0.14 \%$ (db) Trp.

\section{DISCUSSION}

In this study, an in vitro digestion on human mother's milk was performed to simulate the infant's actual digestion. We recovered free Trp from the digested milk and found that it is a potent antioxidant in crude fractions, which were purified by HPLC separation and analyzed by tandem mass spectrometry. The high concentration of Trp in fraction 23 indicates that this amino acid may be contributing to the high antioxidant activity of this fraction.

HM contains a relatively higher content of Trp than do both soy- and milk-based infant formulas (24). Trp concentration of HM protein is about $2.2-2.4 \%$ compared with $1.3 \%$ in cow milk protein and approximately $1.7 \%$ in whey proteinsupplemented infant formulas (25). The difference in Trp content of $\mathrm{HM}$ and infant formula is mainly due to the $\alpha$-lactalbumin portion of whey protein. Cow milk has a wheycasein ratio of approximately 20:80, which is distinctly different from the 60:40 whey-casein ratio in HM (26). Milkbased infant formulae are produced by adding whey protein concentrate to skimmed cow's milk powder to yield a similar whey to casein ratio as that of HM. This modification results in an amino acid composition that is still quite different from that of HM protein, especially in terms of the Trp content (27). Trp is a precursor in the synthesis of the neurotransmitter serotonin, which is involved in the regulation of appetite, circadian rhythm, and affective reaction control (28). The metabolite of Trp, 5-hydroxy-Trp increases growth hormone (29) and stimulates the motility of the gastrointestinal tract. Serotonin synthesis is directly dependent on the availability of the specific precursor (Trp) and on the nutritional status of individuals (30). Serotonin can, in turn, be used for the synthesis of the vitamin nicotinic acid (B3), which prevents pellagra (25).

Trp is a limiting amino acid in human nutrition and to increase its concentration in the infant diet, $\alpha$-lactalbuminenriched formulae may be given to term infants (31). Plasma Trp concentrations of infants fed these enriched formulae do not differ from those of breastfed infants (31). A number of other studies have supplemented free Trp to formulas. Steinberg et al. (32) fed healthy term infants formulae supplemented with 294,588 , and $882 \mu \mathrm{M} / \mathrm{L}$ of free Trp and reported shorter sleep latency in the Trp-fortified formula groups. In a randomized intervention trial, Raiha et al. (33) found no difference in the growth patterns between the control group and the group receiving formula fortified with both $\alpha$-lactalbumin and free Trp. Fazzolari-Nesci et al. (34) also observed that infants fed formulae supplemented with $10 \mathrm{mg}$ Trp/dL had plasma Trp concentrations similar to breastfed infant. Although formulae were supplemented with either $\alpha$-lactalbumin or free Trp in these former studies, the researchers did not measure the antioxidant properties of fortified formulae. Thus, direct comparison with our results is not impossible.

Only one study has evaluated the antioxidant properties of amino acids, including Trp (35). Katayama and Mine analyzed the inhibiting effects of Trp and other amino acids on $\mathrm{H}_{2} \mathrm{O}_{2}-$ induced IL-8 secretion in $\mathrm{CaCO}_{2}$ cells. Melatonin, an endogenously produced Trp metabolite was found by another group to be a scavenger of a number oxygen reactive species (hydroxyl radical, singlet oxygen, and hydrogen peroxide) and nitrogen reactive species (nitric oxide and peroxynitrite anion), when analyzed in vitro and in vivo (36). The finding from the study of Katayama and Mine (35) complements the ORAC assay and reinforces the need to further examine Trp for its ability to reduce oxidative stress, particularly in infants.

Hernandez-Ledesma et al. (23) performed in vitro digestion of $\mathrm{HM}$ and formulas and identified 23 peptides, including HNPI and PLAPLQA, which we also recovered from fraction 23 in this study. Eleven of those peptides were also evaluated for angiotensin-converting enzyme inhibitor activity and antioxidant activity, using the ABTS assay (19). However, the peptide HNPI was not included in these tests, so that the scavenging activity of this peptide according to ORAC and ABTS assays cannot be compared. As in this study, no antioxidant activity was observed for PLAPQA in the ABTS assay (23).

The Trp released from digested HM, as assessed through an in vitro model of a preterm infant's gastrointestinal tract, was identified as a potent radical-scavenging molecule. Its structure contains a ring that can stabilize radicals through resonance or delocalization (Fig. 4), thus enabling it to break radical chain reactions. Although two others amino acids, namely tyrosine (Tyr) and phenylalanine (Phe), also possess ring structures, they were not detected in PF23 in this study. However, Tyr may still be present in the fractions with lower activities so further testing is warranted.

Little work has been performed on the interaction of Trp with other pro- or antioxidants in either the infant diet or the 


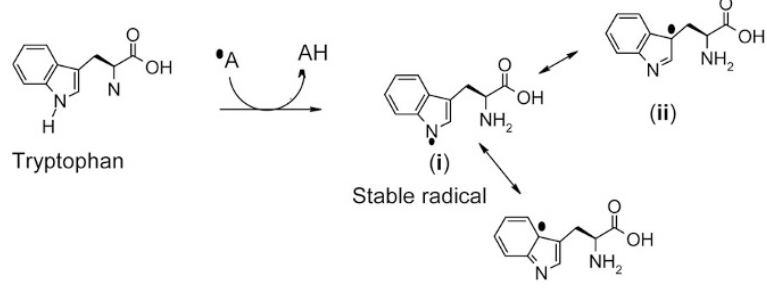

(iii)

Figure 4. Resonance forms of the Trp radical. (i) Trp is a stable radical because its unpaired electron is delocalized (e.g., associated with more than two atoms) or because it has resonance forms (i-iii).

infant metabolism. Katayama and Mine (35) reported that a key antioxidant enzyme, glutathione S-transferase, was induced with Trp pretreatment in a cell culture model. Pazos et al. (37) reported that Trp is the amino acid with the highest antiradical activity. In a rat study, Feksa et al. (38) reported that Trp, vitamin $\mathrm{C}$, and vitamin $\mathrm{E}$ interacted to modulate the oxidant effects of Trp. Clearly, more work needs to be done in this area because HM is rich with many factors that interact to modulate effects seen one molecule at a time.

In conclusion, our results indicate that the amino acid Trp may have a function beyond protein quality and the synthesis of important molecules, including serotonin, melatonin, and niacin. Trp is a powerful free radical scavenger that should be examined further for its affinity to reduce oxidative stress, especially in preterm infants whose endogenous antioxidant defense systems are immature at birth.

Acknowledgment. Technical assistance was provided by Haifeng Yang.

\section{REFERENCES}

1. Halliwell B, Gutteridge JM 1999 Oxidative stress: adaptation, damage, repair and death. In: Free Radicals in Biology and Medicine. 3rd ed. Oxford University Press, Oxford, pp 246-349

2. Tsopmo A, Friel JK 2007 Human milk has anti-oxidant properties to protect premature infants. Curr Pediatric Rev 3:45-51

3. Buonocore G, Perrone S, Longini M, Vezzosi P, Marzocchi B, Paffetti P, Bracci R 2002 Oxidative stress in preterm neonates at birth and on the seventh day of life. Pediatr Res 52:46-49

4. Bancalari E, Wilson-Costello D, Iben SC 2005 Management of infants with bronchopulmonary dysplasia in North America. Early Hum Dev 81:171-179

5. Buonocore G, Perrone S, Longini M, Terzuoli L, Bracci R 2000 Total hydroperoxide and advanced oxidation protein products in preterm hypoxic babies. Pediatr Res 47:221-224

6. Perrone S, Bracci R, Buonocore G 2002 New biomarkers of fetal-neonatal hypoxic stress. Acta Paediatr Suppl 91:135-138

7. Saugstad OD 1988 Hypoxanthine as an indicator of hypoxia: its role in health and disease through free radical production. Pediatr Res 23:143-150

8. Blackburn S 2005 Free radicals in perinatal and neonatal care, part 1: the basics. J Perinat Neonatal Nurs 19:298-300

9. Perrone S, Salvi G, Bellienei CV, Buettner GR 2007 Oxidative stress and nutrition in the preterm newborn. J Pediatr Gastroenterol Nutr 45:S178-S182

10. Kitts DD 2005 Antioxidant phytochemicals and potential synergistic activities at reducing risk of cardiovascular disease. In: Thompson L, Ward W (eds). Food Drug Synergy and Safety. CRC Press LLC, Boca Raton, FL, pp 27-62

11. Autor AP, Frank L, Roberts RJ 1976 Developmental characteristics of pulmonary superoxide dismutase: relationship to idiopathic respiratory distress syndrome. Pediatr Res 10:154-158
12. Frank L, Sosenko IR 1987 Development of lung antioxidant enzyme system in late gestation: possible implications for the prematurely born infant. J Pediatr 110:9-14

13. L'Abbe MR, Friel JK 2000 Superoxide dismutase and glutathione peroxidase content of human milk from mothers of premature and full-term infants during the first 3 months of lactation. J Pediatr Gastroenterol Nutr 31:270-274

14. Friel JK, Martin SM, Langdon M, Herzberg GR, Buettner GR 2002 Milk from mothers of both premature and full-term infants provides better antioxidant protection than does infant formula. Pediatr Res 51:612-618

15. Aycicek A, Erel O, Kocyigit A, Selek S, Demirkol MR 2006 Breast milk provides better antioxidant power than does formula. Nutrition 22:616-619

16. Shehadeh N, Aslih N, Shihab S, Werman MJ, Sheinman R, Shamir R 2006 Human milk beyond one year post-partum: lower content of protein, calcium, and saturated very long-chain fatty acids. J Pediatr 148:122-124

17. Etcheverry P, Wallingford JC, Miller DD, Glahn RP 2004 Calcium, zinc, and iron bioavailabilities from a commercial human milk fortifier: a comparison study. J Dairy Sci 87:3629-3637

18. Jovani M, Barbera R, Farre R, de Aguilera EM 2001 Calcium, iron, and zinc uptake from digests of infant formulas by caco-2 cells. J Agric Food Chem 49:3480-3485

19. Omari TI, Davidson GP 2003 Multipoint measurement of intragastric $\mathrm{pH}$ in healthy preterm infants. Arch Dis Child Fetal Neonatal Ed 88:F517-F520

20. Huang D, Ou B, Hampsch-Woodill M, Flanagan JA, Prior RL 2002 High-throughput assay of oxygen radical absorbance capacity (ORAC) using a multichannel liquid handling system coupled with a microplate fluorescence reader in 96-well format. J Agric Food Chem 50:4437-4444

21. Li W, Hosseinian FS, Tsopmo A, Friel JK, Beta T 2009 Evaluation of antioxidant capacity and aroma quality of breast milk. Nutrition 25:105-114

22. Wisniewski R, Boschetti E, Jungbauer A 1996 Process design for large-scale chromatography of biomolecules. In: Avis KE, Wu VL (eds). Biotechnology and Biopharmaceutical Manufacturing, Processing, and Preservation. CRC Press, Buffalo Grove, IL, pp 61-181

23. Hernandez-Ledesma B, Quiros A, Amigo L, Recio I 2007 Identification of bioactive peptides after digestion of human milk and infant formula with pepsin and pancreatin. Int Dairy J 17:42-49

24. Sarwar G, Botting HG, Peace RW 1989 Amino-acid rating method for evaluating protein adequacy of infant formulas. J Assoc Off Anal Chem 72:622-626

25. Heine WE 1999 The significance of tryptophan in infant nutrition. Adv Exp Med Biol 467:705-710

26. Stinnakre MG, Vilotte JL, Soulier S, Mercier JC 1994 Creation and phenotypic analysis of alpha-lactalbumin-deficient mice. Proc Natl Acad Sci USA 91:65446548

27. Lonnerdal B, Chen CL 1990 Effects of formula protein level and ratio on infant growth, plasma amino-acids and serum trace-elements 1 . Cows milk formula. Acta Paediatr Scand 79:257-265

28. Heine W, Radke W, Wutzke KD, Peters E, Kundt G 1996 Alpha-lactalbuminenriched low-protein infant formulas: a comparison to breast milk feeding. Acta Paediatr 85:1024-1028

29. Lancranjan I, Wirzjustice A, Puhringer W, Pozo ED 1977 Effect of 1-5 hydroxytryptophan infusion on growth-hormone and prolactin secretion in man. J Clin Endocrinol Metab 45:588-593

30. Goodwin GM, Fairburn CG, Cowen PJ 1987 The effects of dieting and weight loss on neuroendocrine responses to tryptophan, clonidine, and apomorphine in volunteers: important implications for neuroendocrine investigations in depression. Arch Gen Psychiatry 44:952-957

31. Sandstrom O, Lonnerdal B, Graverholt G, Hernell O 2008 Effects of alphalactalbumin-enriched formula containing different concentrations of glycomacropeptide on infant nutrition. Am J Clin Nutr 87:921-928

32. Steinberg LA, O'Connell NC, Hatch NF, Picciano MF, Birch LL 1992 Tryptophan intake influences infants' sleep latency. J Nutr 122:1781-1791

33. Raiha NC, Fazzolari-Nesci A, Cajozzo C, Puccio G, Monestier A, Moro G, Minoli I, Haschke-Becher E, Bachmann C, Van't Hof M, Carrie Fassler AL, Haschke F 2002 Whey predominant, whey modified infant formula with protein/energy ratio of $1.8 \mathrm{~g} / 100 \mathrm{kcal}$ : adequate and safe for term infants from birth to four months. J Pediatr Gastroenterol Nutr 35:275-281

34. Fazzolari-Nesci A, Domianello D, Sotera V, Raiha NC 1992 Tryptophan fortification of adapted formula increases plasma tryptophan concentrations to levels not different from those found in breast-fed infants. J Pediatr Gastroenterol Nutr $14: 456-459$

35. Katayama S, Mine Y 2007 Antioxidative activity of amino acids on tissue oxidative stress in human intestinal epithelial cell model. J Agric Food Chem 55:8458-8464

36. Reiter RJ, Tan D, Mayo JC, Sainz RM, Leon J, Czarnocki Z 2003 Melatonin as an antioxidant: biochemical mechanisms and pathophysiological implications in humans. Acta Biochim Pol 50:1129-1146

37. Pazos M, Andersen ML, Skibsted LH 2006 Amino acid and protein scavenging of radicals generated by iron/hydroperoxide system: an electron spin resonance spin trapping study. J Agric Food Chem 54:10215-10221

38. Feksa LR, Latini A, Rech VC, Wajner M, Dutra-Filho CS, de Souza Wyse AT, Wannmacher CM 2006 Promotion of oxidative stress by L-tryptophan in cerebral cortex of rats. Neurochem Int 49:87-93 